\title{
Characterization of erythrovirus B19 genomes isolated in liver tissues from patients with fulminant hepatitis and biliary atresia who underwent liver transplantation
}

\author{
1. Department of Pathology, National Institute of Infectious Diseases, Tokyo, Japan \\ 2. Department of Transplantation Surgery, Nagoya University Hospital, Aichi, Japan \\ 3. Department of Transplantation Surgery, Kyoto University Hospital, Kyoto, Japan \\ 4. Institute of Biomedical Research and Innovation, Hyogo, Japan \\ 5. Department of Surgery, International Medical Center of Japan, Tokyo, Japan \\ 6. Aiba Clinic, Saitama, Japan
}

Kenji Abe ${ }^{1}$, Tetsuya Kiuchi ${ }^{2}$, Koichi Tanaka 3,4, Yoshihiro Edamoto ${ }^{5}$, Naoto Aiba 6 and Tetsutaro Sata ${ }^{1}$

Correspondence to: Dr. Kenji Abe, Department of Pathology, National Institute of Infectious Diseases, Toyama 1-23-1, Shinjuku-ku, Tokyo 162-8640, JAPAN. TEL: (81) 3-5285-1111 ext. 2624 FAX: (81) 3-5285-1189 E-mail: kenjiabe@nih.go.jp

Received: 2007.02.20; Accepted: 2007.04.05; Published: 2007.04.05

Background: Fulminant hepatitis and biliary atresia are serious problems and their causes have not been explained well. We investigated whether or not erythrovirus B19 is a candidate etiologic agent in such liver disease patients who had undergone liver transplantation.

Methods: Liver tissues from 47 patients consisted of 28 fulminant hepatitis and 19 biliary atresia were examined to detect B19 genes by PCR and further analyzed their genomic characterization.

Results: B19 DNA was detected by nested PCR in 10 of 28 cases (35.7\%) livers in the fulminant hepatitis group and 7 of $19(36.8 \%)$ livers in the biliary atresia group, respectively (statistically not significant). Importantly, among the 8 hepatic B19 DNA-positive patients who had paired samples of liver and serum, the serum B19 genome was detectable in only one case. B19 mRNA was identified in all of 10 fulminant hepatitis cases with hepatic B19 DNA, but only 1 out of $7(14.3 \%)$ cases in biliary atresia tested. Furthermore, we obtained ten isolates having the B19 genome with nearly full-length sequences. Interestingly, phylogenetic analysis based on the NS1 gene revealed three different clusters: two for isolates from fulminant hepatitis and the other for isolates from biliary atresia.

Conclusions: Our results presented here suggested that B19 may be an etiologic agent of fulminant hepatitis.

Key words: Erythrovirus B19; erythrovirus B19 genome; fulminant hepatitis; biliary atresia; liver transplantation

\section{Introduction}

Erythrovirus (formally parvovirus) B19 (B19) causes a wide range of diseases in humans. B19 is a currently the only accepted member of the Erythrovirus genus and the only erythrovirus known to be pathogenic in humans. Several workers reported cases of children with fulminant or acute hepatitis with acute B19 infection and suggested that B19 could be the cause of the hepatitis [1-3]. Their diagnosis was based on the detection of the serum B19 DNA by PCR assay. However, the cause of fulminant hepatitis and biliary atresia in many patients is unexplained and this is a serious issue, especially in newborns and infants. More than $80 \%$ of these cases ultimately require liver transplantation or die of hepatic failure. B19 has been proposed as the cause of the fulminant hepatic failure in patients with or without aplastic anemia, on the basis of the presence of B19 DNA in liver specimens. However, the mechanism of liver damage by B19 infection remains conjectural. An immunologi- cally-mediated mechanism of B19-induced hepatocyte destruction has been postulated, but not documented. Thus, there could be an unsuspected cofactor that is associated with B19 infection and is deleterious to the liver. To address these important issues, we conducted a molecular characterization of B19 genomes isolated from patients with fulminant hepatitis and biliary atresia.

\section{Materials and Methods}

Patients: We compared 2 different groups consisted with 47 Japanese patients (aged from newborns to 66 years old; 24 males and 23 females). Group A consisted of 28 patients with fulminant hepatitis (14 males and 14 females; 9 infants less than one year old, 8 children 1-10 years old, 3 adolescents $11-20$ years old and 8 adults over 21 years old). On the other hand, group B consisted of 19 patients with biliary atresia; 7 males and 12 females; 4 infants less than one year old, 10 children 1-10 years old, 4 adolescents 11-20 years old and one adult over 21 years old). All patients ex- 
amined had no evidence of hepatitis virus infections and diagnosed as non- $\mathrm{B}$, non-C hepatitis. Most of the patients tested underwent living-donor liver transplantation at Kyoto University Hospital from 1994 to 2000. Majority of patients had history of blood transfusion or blood product infusion in their course of treatment. The liver tissues and serum samples from recipients obtained at operation stored at $-80^{\circ} \mathrm{C}$ until used. Informed consent for participation in this study was obtained from each individual.

Nucleic acid extraction, PCR and sequencing analysis: Nucleic acids (DNA/RNA) were extracted from frozen liver tissues and serum using SepaGene RV-R Kit (Sanko-Junyaku, Tokyo, Japan). The resulting pellet was resuspended in $100 \mu \mathrm{l}$ RNase-free water, following the manufacturer's instruction. B19 DNA was screened by nested PCR using primers designed from VP1 region; PV1 (sense, 5'-GCT GTT AAG GAT GTT ACA GA-3', nucleotide (nt) 3520-3521) and PV1R (antisense, 5'-GGA TCC GTA TAA GGG ATT GT-3', nt 3882-3901) for the 1st PCR and PV2 (sense, 5'-CAG GTT ACT GAC AGC ACT AC-3', nt 3541-3560) and PV2R (antisense, 5'-TGT TGA CTG CAG CCC TCT AA-3', nt 3848-3867) for the 2nd PCR (Yoto et al., 1996). The sensitivity of this PCR assay allowed detection of as few as 10 copies of B19 genome. In addition, we sequenced the B19 isolates consisted of $4561 \mathrm{bp}$ covering NS1, VP1 and VP2 regions obtained from liver tissues of patients. For this purpose, three overlapping PCR products (fragments $A$ to $C$ ) were generated; for fragment A (1944 bp), PV3 (sense, 5'-TTT CCC GCC TTA TGC AAA TGG GCA G-3', nt 393-417) and PV5R (antisense, 5'-AGC TCC CAC ATG GCA GCT AC-3', nt 2533-2552) for the 1st PCR and PV4 (sense, 5'-TGT AAC GGT TAA AAT GGG CGG AGC G-3', nt 457-481) and PV6R (antisense, 5'- CCC CTT ACA CCG TCC CAC AC-3', nt 2382-2401) for the 2nd PCR; for fragment B (1835 bp), PV5 (sense, 5'-GCA GCA GTG GTG GTG AAA GC-3', nt 2143-2162) and PV1R for the 1st PCR and PV6 (sense, 5'-GGC GCC TGG AAC ACT GAA AC-3', nt 2208-2227) and PV2R for the 2nd PCR; for fragment C (1297 bp), PV1 and PV3R (sense, 5'-TAC AGT CTG GGT GGT ACT GGT GGG C-3', nt 5010-5034) for the 1st PCR and PV2 and PV4R (antisense, 5'-CTG GTG GGC GTT TAG TTA CGC ATC C-3', nt 4994-5018). Nucleotide positions are based on B19-HV isolate (accession No. AF162273). The PCR was done with AmpliTaq Gold (Perkin Elmer, Norwalk, Conn.) and Blend-Taq-Plus DNA polymerase (TOYOBO Co., Tokyo, Japan). The amplicons were separated by $1 \%$ agarose gel electrophoresis and purified using the QIAquick gel extraction kit (Qiagen Inc., Chatsworth, Calif.). Recovered amplicons were subjected to direct sequencing from both directions using the ABI PRISMTM Big Dye Terminator Cycle Sequencing Ready Reaction Kit (Perkin Elmer). Sequences of amplified DNA were determined using a sequencer (ABI model 373A; Applied Biosystems, Foster City, Calif.). Additionally, hepatitis A, B, C and E genomes were also determined by the nested PCR.

Detection of B19 mRNA: The purified RNA ob- tained from liver tissues as described above, was digested by RQ I DNase (Promega Co., Madison, Wis.) to remove any DNA. B19 cDNA was synthesized from the pretreated RNA by reaction with 100 units of Moloney murine leukemia virus reverse transcriptase (Promega Co.) and PV1R antisense primer. The cDNA obtained was subjected to nested PCR amplification.

Phylogenetic analysis: Characterization of B19 isolates was examined by the phylogenetic analysis as described previously [4]. The data were analyzed using a standard statistical software package (Stat View; Brain Power Inc., Calabases, CA, U.S.A.).

Statistic analyses: Statistic analyses were performed by the chi-square test. A difference with a $P$ value of less than 0.05 was considered significant.

\section{Results}

B19 DNA in liver tissues was detected in 10 of 28 (35.7\%) patients with fulminant hepatitis and 7 of 19 $(36.8 \%)$ patients with BA, respectively (statistically not significant). Except for two infants with fulminant hepatitis who were HBV DNA-positive, all cases tested were negative for HAV RNA, HCV RNA and HEV RNA. Importantly, among the hepatic B19 DNA-positive 8 patients who had paired samples of liver tissues and serum, the serum B19 sequence was detectable in only one case (Table 1 ).

Table 1. Comparison of B19 DNA detection between serum and liver tissue

\begin{tabular}{|c|c|c|c|c|}
\hline \multirow{2}{*}{\multicolumn{2}{|c|}{ Case \# Age/Sex }} & \multirow[b]{2}{*}{ Diagnosis } & \multicolumn{2}{|c|}{ B19 DNA } \\
\hline & & & Serum & Liver \\
\hline 23 & $15 y / M$ & Fulminant hepatitis & - & + \\
\hline 28 & $9 \mathrm{y} 4 \mathrm{~m} / \mathrm{F}$ & Fulminant hepatitis & - & + \\
\hline 30 & $3 \mathrm{~m} / \mathbf{M}$ & Fulminant hepatitis & - & + \\
\hline 34 & $26 y / F$ & Fulminant hepatitis & - & + \\
\hline 87 & $39 y / F$ & Fulminant hepatitis & - & + \\
\hline 66 & $1 \mathrm{y} 5 \mathrm{~m} / \mathrm{M}$ & Fulminant hepatitis & + & + \\
\hline 107 & $9 \mathrm{~m} / \mathbf{M}$ & Fulminant hepatitis & - & + \\
\hline 108 & $41 \mathrm{y} / \mathrm{F}$ & Fulminant hepatitis & - & + \\
\hline 13 & $5 \mathrm{~m} / \mathrm{M}$ & Fulminant hepatitis & NA & + \\
\hline 18 & $1 \mathrm{y} 10 \mathrm{~m} / \mathrm{F}$ & Fulminant hepatitis & NA & + \\
\hline
\end{tabular}

To determine the form of viral replication, the presence of B19 mRNA in the liver tissue was examined. The results showed that B19 mRNA was present in all cases with fulminant hepatitis to be hepatic B19-positive, but only 1 of 7 (14.3\%) cases in biliary atresia tested. Amplification of B19 DNA did not take place in the absence of reverse transcriptase, indicating minimal DNA contamination.

In addition to the above-described examinations, to characterize the B19 genomes, we obtained 14 isolates of the B19 genome (10 isolates with nearly full-length sequences (4561 bp) that covered the complete genes of NS1, VP1 and VP2 and 4 isolates with only one short sequence in the NS1). All B19 isolates showed an overall identity of $98 \%$ at the nt level among each isolate with $4561 \mathrm{bp}$ recovered in this study and to prototypes of B19 HV (F162273) and B19 
$\mathrm{Au}$ (M13178), and an identity of $87 \%$ to the B19 V9 isolate (AY083234). Interestingly, phylogenetic analysis based on the NS1 gene revealed three different clusters: two (genotypes 1 and 3) for isolates from fulminant hepatitis and the other (genotype 2) for isolates from biliary atresia (Fig. 1). Among each genotype, specific sites of the deduced amino acid sequence change were identified in the NS1 gene (Fig. 2). In 7 isolates belonging to group 2, the amino acid at position 181 and 201 showed methionine and aspartic acid, respectively. Interestingly, aspartic acid substitution in 201 localized within the putative B cell epitope. Furthermore, in 5 out of 6 isolates belonging to group 3 , the amino acid at position 183 substituted to alanine.

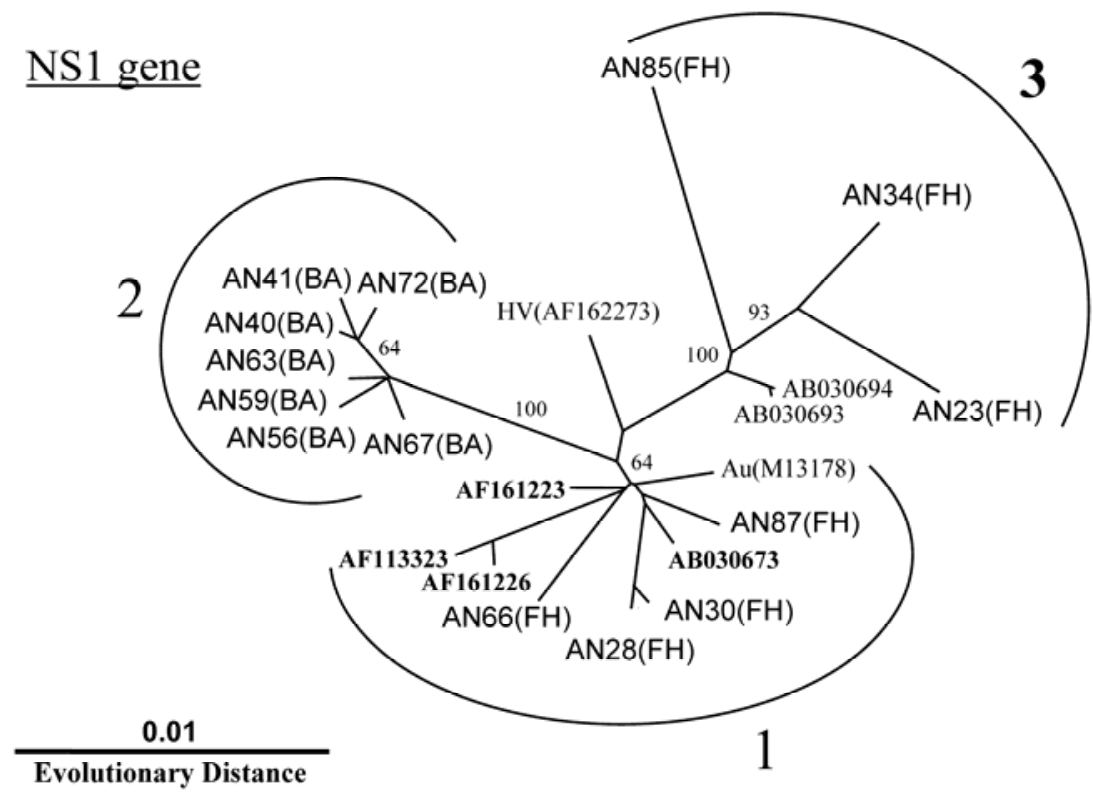

Figure 1. Phylogram generated by neighbor-joining analysis of genetic distances in the NS1 region of erythrovirus B19. The percentage of bootstrap replicates supporting these branches is shown. FH=fulminant hepatitis, BA=biliary atresia.

\section{Genotype}

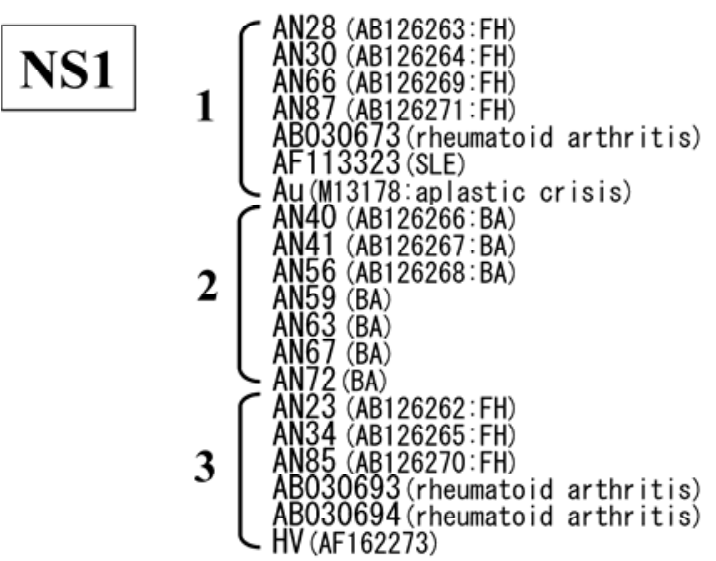
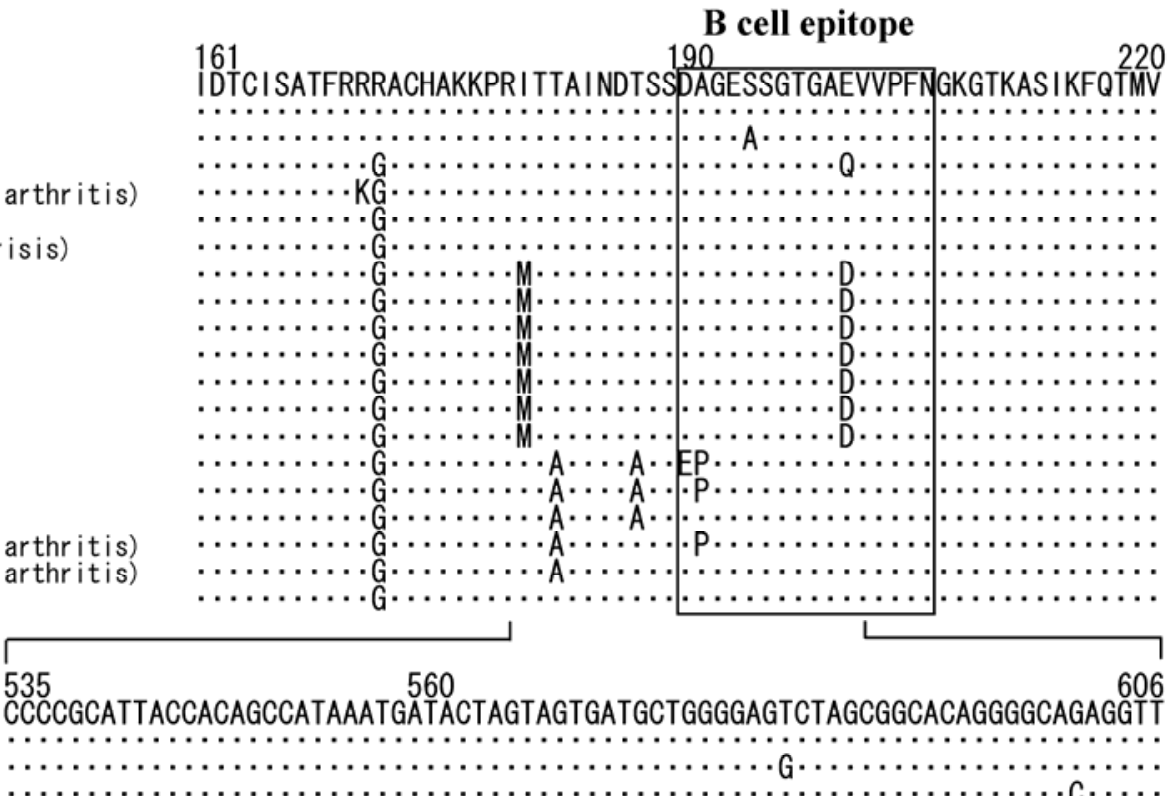

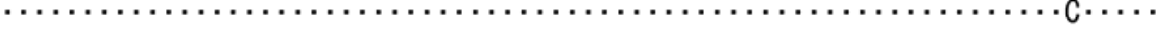

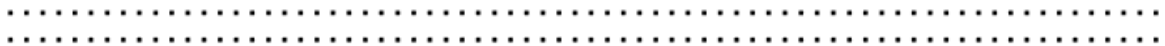
. . .

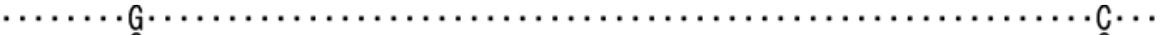

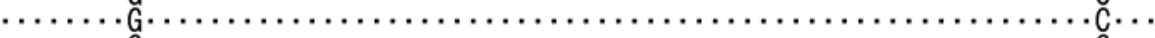

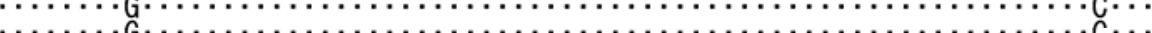

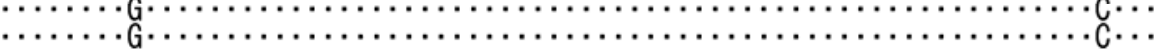

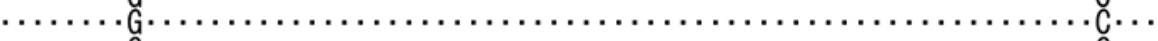

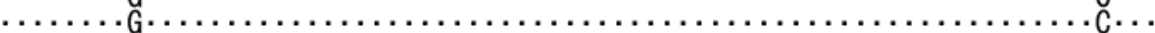

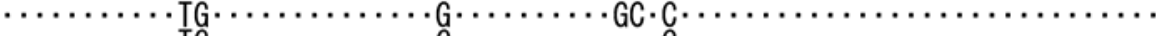

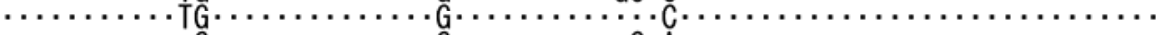

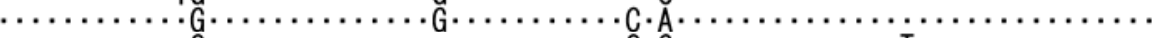

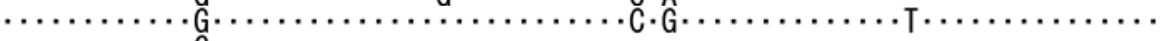

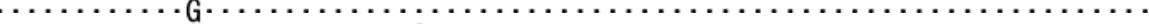

Figure 2. Alignment of amino acid sequences (upper) and nucleic acid sequences (lower) in the NS1 region of erythrovirus B19. Dot (.) indicate amino acids identical to the top sequence. B cell epitope region indicated by box. 
Nucleotide sequence data reported are available in the DDBJ/GenBank/EMBL database under the accession numbers: AB126262 for B19-AN23, AB126263 for B19-AN28, AB126264 for B19-AN30, AB126265 for B19-AN34, AB126266 for B19-AN40, AB126267 for B19-AN41, AB126268 for B19-AN56, AB126269 for B19-AN66, AB126270 for B19-AN85 and AB126271 for B19-AN87.

\section{Discussion}

The etiology of hepatitis remains obscure in $3-10 \%$ of cases in Europe [5] and the United States [6] and up to $30 \%$ of cases in Asia $[7,8]$. Particularly, the cause of fulminant hepatitis in children is unexplained in up to $50 \%$ of cases in our hospital. Once well-known hepatotropic agents and metabolic, toxic, and immunological causes have been excluded, an infectious origin remains a possibility. Opportunistic infections may be the cause of fulminant hepatitis in immunocompromised patients.

On the other hand, the concept that biliary atresia consists of two major types based on the clinical aspects has been accepted $[9,10]$. One is the fetal, embryonic or prenatal type, and the other is the acquired or perinatal type. The perinatal type is believed to account for $70-80 \%$ of all biliary atresia. The etiology of biliary atresia has remained obscure. Two hypotheses have been put forward. The first is called the ductal plate malformation theory, which assumes a congenital anomaly of the intrahepatic bile trees. According to the other theory, the progressive occlusion of the extrahepatic bile duct is probably triggered by viral infection and worsened by the subsequent immunopathological process. Reovirus, rotavirus [11, 12], cytomegalovirus $[13,14]$ and others have been proposed as likely causative viruses, but no clear relation has been found. To the best of our knowledge, there are only a few reports $[1,15,16]$ on the analysis of viral infections based on direct detection of viral genomes from liver tissues.

B19 was discovered serendipitously in the sera of normal blood bank donors while screening for the hepatitis B virus. B19, a member of the family Parvoviridae, subfamily Parvovirinae, genus Erythrovirus, is a small single stranded DNA virus. B19 is a pathogenic virus that is widely distributed in human populations. This virus is responsible for various clinical manifestations including erythema infectiosum in children, polyarthropathy syndrome in adults, transient aplastic crisis inpatients with chronic anemia, persistent infections manifesting as chronic anemia in immunocompromised patients, transplant recipients, and hydrops fetalis and fetal death by intrauterine infection. Aplastic anemia, which occurs in 33\% of children and $5 \%$ of adult patients who undergo orthotopic liver transplantation (OLT) for non-A, non-B, non-C fulminant hepatitis is pointed out as a complication with a high rate of mortality [17]. The cause of aplastic anemia after OLT may include factors such as posthepatitic aplasia, B19, and drugs. Langnas et al. [1] reported the possibility of B19 as the cause of non-A-C fulminant hepatitis-associated aplastic anemia. Actually, B19 may be an etiologic agent for hepatitis-associated aplastic anemia, because of the known tropism of B19 for erythroid precursors [18]. On the other hand, negative data are also present. Wong et al. [16] reported no association with fulminant hepatitis or hepatitis-associated aplastic anemia. Lee et al. [19] reported no evidence for hepatitis E or parvovirus B19 infection in patients with acute liver failure.

To investigate the possibility that B19 infection of the liver might cause fulminant hepatitis and biliary atresia, we conducted a study to obtain direct evidence of a viral sequence in the liver from patients who underwent liver transplantation. In this retrospective study of 47 patients with liver diseases of unknown etiology, B19 DNA was detected in the liver obtained from 17 patients. We postulated that the virus might even be detected in liver that is serologically negative for the virus. In fact, our results showed that only one case was sero-positive for the B19 sequence among 8 cases with intrahepatic-B19 DNA. These results suggest that seronegativity for the B19 sequence does not exclude the existence of present infection of B19 within the liver. Similar finding was reported by Eis-Hubinger et al [20]. They showed B19 DNA is frequently present in livers of anti-B19 seropositive adults suggesting persistence of B19 in the liver. Our results also indicate that direct detection of viral genomes in tissues by a highly sensitive method is as important as serology. In the present study, we found a high prevalence of B19 infection not only in fulminant hepatitis patients, but also in biliary atresia patients. However, all fulminant hepatitis patients infected with B19 had the replicative form of B19 mRNA. While, low rate of B19 mRNA detection in biliary atresia patients who had intra-hepatic B19 DNA suggested silent infection of B19. This suggests that B19 may be a cause of severe hepatic diseases although this remains to be investigated.

B19 has a genome length of $5.4 \mathrm{~kb}$ with hairpin structures at each extremity. B19 encodes three major viral proteins: VP1 and NP2, the viral capsid proteins, and NS1, a nonstructural protein. Both VP1 and VP2 are derived from overlapping reading frames and share substantial amino acid sequences. NS1 is known to be implicated in viral replication, the activation of viral gene transcription, and target cell cytotoxicity [21-25]. Among the sequence records deposited in the database, only 16 isolates of B19 have been sequenced in the full or nearly full-genome so far. Here we reported that the nearly full-length sequence of B19 was isolated from 10 patients with liver diseases. It is known that B19 has a genome length of $5.4 \mathrm{~kb}$ with hairpin structures at each extremity; in addition, a partial deletion and rearrangement of the sequence exist in the extremity regions. These findings indicate the difficulty in amplifying the sequences in these regions. In our study, we attempted to amplify each extremity, but failed. Interestingly, phylogenetic analysis based on the NS1 gene revealed three different clusters: two for isolates from fulminant hepatitis and the 
other for isolates from biliary atresia cases. This finding suggests the existence of genotypes. Viral genotype has diagnostic and clinical implications, including use for assessing responses to anti-viral therapy and future vaccine development. The possibility that such variation is involved in the severity of associated liver disease, and that the difference between symptomatic and asymptomatic infection, may also occur as well as HBV and HCV. Furthermore, among each genotype, specific sites of the deduced amino acid sequence change were identified in the NS1 gene. Clarification of the relation between the genotypes or variants of B19 and its pathogenicity in hepatic failure including hepatitis and biliary atresia is awaited with great interest. To solve these important issues, a geographical study of the B19 genomes and various diseases including liver diseases in different countries is now planning and we shall report on further investigations in the future.

In conclusion, we found a high prevalence of B19 infection in livers from patients with fulminant hepatitis and biliary atresia. Interestingly, all fulminant hepatitis patients infected with B19 had the replicative form of B19 mRNA. It is noteworthy that serum B19 DNA was not detected in 7 of 8 cases that were hepatic B19 DNA-positive. Phylogenetic analysis based on the NS1 gene revealed three different clusters. Our results presented here suggested that B19 may serve as an etiologic agent for severe hepatitis.

\section{Conflict of interest}

The authors have declared that no conflict of interest exists.

\section{References}

1. Langnas AN, Markin RS, Cattral MS, et al. Parvovirus B19 as a possible causative agent of fulminant liver failure and associated aplastic anemia. Hepatology 1995; 22: 1661-5

2. Sokal EM, Melchior M, Cornu C, et al. Acute parvovirus B19 infection associated with fulminant hepatitis of favourable prognosis in young children. Lancet 1998; 352: 1739-41

3. Yoto Y, Kudoh T, Haseyama K, et al. Human parvovirus B19 infection associated with acute hepatitis. Lancet 1996; 347: 868-9

4. Abe K, Inami T, Ishikawa $\mathrm{K}$, et al. TT virus infection in nonhuman primates and characterization of the viral genome: Identification of simian TT virus isolates. J Virol. 2000; 74: 1549-53.

5. Romano L, Fabris P, Tanzi E, et al. GBV-C/hepatitis G virus in acute nonA-E hepatitis and in acute hepatitis of defined aetiology in Italy. J Med Virol. 2000; 61: 59-64

6. Alter MJ, Gallagher M, Morris TT, et al. Acute non-A-E hepatitis in the United States and the role of hepatitis G virus infection. Sentinel Counties Viral Hepatitis Study Team. N Engl J Med. 1997; 336: 741-6

7. Chu CM, Lin SM, Hsieh SY, et al. Etiology of sporadic acute viral hepatitis in Taiwan: the role of hepatitis $C$ virus, hepatitis $E$ virus and $G B$ virus- $C$ /hepatitis $G$ virus in an endemic area of hepatitis A and B. J Med Virol. 1999; 58: 154-9

8. Sato S, Suzuki K, Takikawa Y, et al. Japanese National Study Group of Fulminant Hepatitis. Clinical epidemiology of fulminant hepatitis in Japan before the substantial introduction of liver transplantation: an analysis of 1309 cases in a 15-year national survey. Hepatol Res. 2004; 30: 155-61
9. Balistreri WF, Grand R, Hoofnagle JH, et al. Biliary atresia: current concepts and research directions. Summary of a symposium. Hepatology 1996; 6: 1682-92

10. Sokol RJ, Mack C. Etiopathogenesis of biliary atresia. Semin Liver Dis. 2001; 21:517- 24.

11. Bobo L, Ojeh C, Chiu D, et al. Lack of evidence for rotavirus by polymerase chain reaction/enzyme immunoassay of hepatobiliary samples from children with biliary atresia. Pediatr Res. 1997; 41: 229-34

12. Riepenhoff-Talty M, Gouvea V, Evans MJ, et al. Detection of group $\mathrm{C}$ rotavirus in infants with extrahepatic biliary atresia. J Infect Dis. 1996; 174: 8-15

13. Fischler B, Ehrnst A, Forsgren M, et al. The viral association of neonatal cholestasis in Sweden: a possible link between cytomegalovirus infection and extrahepatic biliary atresia. J Pediatr Gastroenterol Nutr. 1998; 27: 57-64

14. Tarr PI, Haas JE, Christie DL. Biliary atresia, cytomegalovirus, and age at referral. Pediatrics 1996; 97: 828-31

15. Karetnyi YV, Beck PR, Markin RS, et al. Human parvovirus B19 infection in acute fulminant liver failure. Arch Virol. 1999; 144: 1713-24

16. Wong S, Young NS, Brown KE. Prevalence of parvovirus B19 in liver tissue: no association with fulminant hepatitis or hepatitis-associated aplastic anemia. J Infect Dis. 2003; 187: 1581-6

17. Cattral MS, Langnas AN, Markin RS, et al. Aplastic anemia after liver transplantation for fulminant liver failure. Hepatology 1994; 20: 813-8

18. Ozawa K, Kurtzman G, Young N. Productive infection by B19 parvovirus of human erythroid bone marrow cells in vitro. Blood 1987; 70: 384-91

19. Lee WM, Brown KE, Young NS, et al. No evidence for parvovirus B19 or hepatitis E virus as a cause of acute liver failure. Dig Dis Sci. 2006; 51:1712-5

20. Eis-Hubinger AM, Reber U, Abdul-Nour T, Glatzel U, Lauschke H, Putz U. Evidence for persistence of parvovirus B19 DNA in livers of adults. J Med Virol. 2001; 65: 395-401

21. Ozawa K, Ayub J, Kajigaya S, et al. The gene encoding the nonstructural protein of B19 (human) parvovirus may be lethal in transfected cells. J Virol. 1988; 62: 2884-9

22. Doerig C, Hirt B, Antonietti JP, et al. Nonstructural protein of parvoviruses B19 and minute virus of mice controls transcription. J Virol. 1990; 64: 387-96

23. Moffatt S, Tanaka N, Tada K, et al. A cytotoxic nonstructural protein, NS1, of human parvovirus B19 induces activation of interleukin-6 gene expression. J Virol. 1996; 70: 8485-91

24. Poole BD, Karetnyi YV, Naides SJ. Parvovirus B19-induced apoptosis of hepatocytes. J Virol. 2004; 78: 7775-83

25. Poole BD, Zhou J, Grote A, Schiffenbauer A, Naides SJ. Apoptosis of liver-derived cells induced by parvovirus B19 nonstructural protein. J Virol. 2006; 80: 4114-21 